\title{
SIMULATED-DATA ADAPTATION BASED PIECEWISE LINEAR TRANSFORMATION FOR ROBUST SPEECH RECOGNITION
}

\author{
Nattanun Thatphithakkul*, Boontee Kruatrachue \\ King Mongkut's Institute of Technology Ladkrabang, Bangkok, 10520, Thailand
}

Chai Wutiwiwatchai, Sanparith Marukatat, and Vataya Boonpiam

National Electronics and Computer Technology Center, Bangkok, 12120, Thailand

Received 12 June 2006

\begin{abstract}
This paper proposes an efficient method of simulated-data adaptation for robust speech recognition. The method is applied to tree-structured piecewise linear transformation (PLT). The original PLT selects an acoustic model using tree-structured HMMs and the acoustic model is adapted by input speech in an unsupervised scheme. This adaptation can degrade the acoustic model if the input speech is incorrectly transcribed during the adaptation process. Moreover, adaptation may not be effective if only the input speech is used. Our proposed method increases the size of adaptation data by adding noise portions from the input speech to a set of prerecorded clean speech, of which correct transcriptions are known. We investigate various configurations of the proposed method. Evaluations are performed with both additive and real noisy speech. The experimental results show that the proposed system reaches higher recognition rate than MLLR, HMM-based model selection and PLT.
\end{abstract}

Keywords: Robust speech recognition, piecewise linear transformation, simulated-data adaptation

\section{INTRODUCTION}

It is commonly known that a speech recognition system trained by speech in a clean or nearly clean environment cannot achieve good performance when working in noisy environment. Research on robust speech recognition is then necessary. Gales [1] has classified the techniques of robust speech recognition into 4 approaches: 1) extraction of robust speech feature, 2) estimation of clean speech, 3) construction of robust model, and 4) combination of three previous techniques. Each of these approaches has both advantages and disadvantages. This paper focuses on the model-based approach, which has achieved good recognition results [1]. The model-based approach aims to create or to adapt the acoustic model in specific environments. Several techniques of model adaptation have been proposed such as maximum likelihood linear regression (MLLR) [2, 3], maximum a posteriori (MAP) adaptation [4, 5], parallel model combination (PMC) [6 - 8], and piecewise linear transformation (PLT) [9, 10].

In this work, we are interested in the adaptation technique of piecewise linear transformation

\footnotetext{
* Corresponding author e-mail: S6060008@kmitl.ac.th
} 
with tree-structured model selection [10, 11], proposed by Zhang, Sugimura and Furui [10]. This technique is based on an unsupervised acoustic model adaptation using the incoming speech. It was proven to be efficient in both accuracy and computational cost compared to the PMC technique [9]. However, a problem of the PLT is that the acoustic model may not be well adapted if the incoming speech is very short as found in most of isolated-word recognition tasks. Moreover, in the unsupervised adaptation, a wrong transcription of the input speech strongly degrades the adapted acoustic model.

Therefore, this paper presents a new adaptation scheme using simulated-data adaptation applied to piecewise linear transformation (PLT). Indeed, the simulated-data adaptation process aims to increase the data used in adaptation by adding the background noise extracted from the current input signal to existing clean speech. Since correct transcriptions of the clean speech are known, using the simulated-data is supervised adaptation. In this paper, both supervised adaptation and semi-supervised adaptation where the input speech is included in the adaptation set are investigated. Selection of a recognition result from the results provided by both the adapted and original acoustic models allows further improvement of the recognition accuracy. It should be noted that this idea of simulated-data adaptation is not limited to PLT adaptation. It can be used in other adaptation algorithms such as the general MLLR process. A comparison between normal MLLR and MLLR using simulated-data adaptation is also investigated.

The proposed system was evaluated with 3 groups of environments. The first group contained a clean environment and 9 types of noisy environments that have been trained in the system. The second group contained other 2 types of noises not trained in the system. Noisy speech was prepared by adding noise signals from JEIDA [12] , NOISEX-92 [13] and an exhibition in Thailand (NAC 2005) to the clean speech taken from NECTEC-ATR Thai speech corpus [14] at various $\operatorname{SNR}(0,10,15 \mathrm{~dB})$. The third group contained speech signal recorded in real environment of another exhibition in Thailand (ICT-EXPO 2005). The estimated SNR for this last group was $0-5 \mathrm{~dB}$.

We will review the PLT algorithm in the next section, followed by an explanation of our proposed models in Section 3. Section 4 describes the data used in these works and experimental results are reported in Section 5. Section 6 concludes this paper with some future works.

\section{PIECEWISE LINEAR TRANSFORMATION (PLT)}

The PLT method [10] is composed of 2 main steps namely the tree-structure HMM construction and the MLLR adaptation for the current input signal. Figure 1 shows a flow diagram of the PLT method. In the first step, a wide variety of noise data were collected and classified into noise clusters using hierarchical clustering. The root node includes all noises and all SNR conditions and each leaf node consists of only one noise at one SNR condition. Intermediate nodes in this tree contain noises from different environments and from different SNR which are similar. A noise-added speech HMM is constructed for each node. Using this tree structure, an unknown noise environment which is similar to combination of known environments should be handled by non-leaf HMM. The resulting tree-structured HMM allows representing both known and some unknown noises. In the recognition phase, the noise-cluster HMM that best fitted the input speech was selected and further adapted to reduce mismatches with the input speech by the MLLR method. In both processes, HMM selection and adaptation using linear transformation are based on the likelihood maximization criterion.

\section{PIECEWISE LINEAR TRANSFORMATION WITH SIMULATED-DATA ADAPTATION}

The piecewise linear transformation with simulated-data adaptation, called S-PLT hereafter, is 
similar to PLT in Figure 1 except that the recognition process is replaced by the procedure shown in Figure 2. The idea of the proposed method is to increase the number of adaptation data using a set of pre-recorded clean speech, of which exact transcriptions are known. A set of adaptation data is simulated by adding noise portions extracted from the input speech to a set of clean speech. Using the existing speech with known transcriptions can be called supervised adaptation. The next subsection explains in details the whole process.

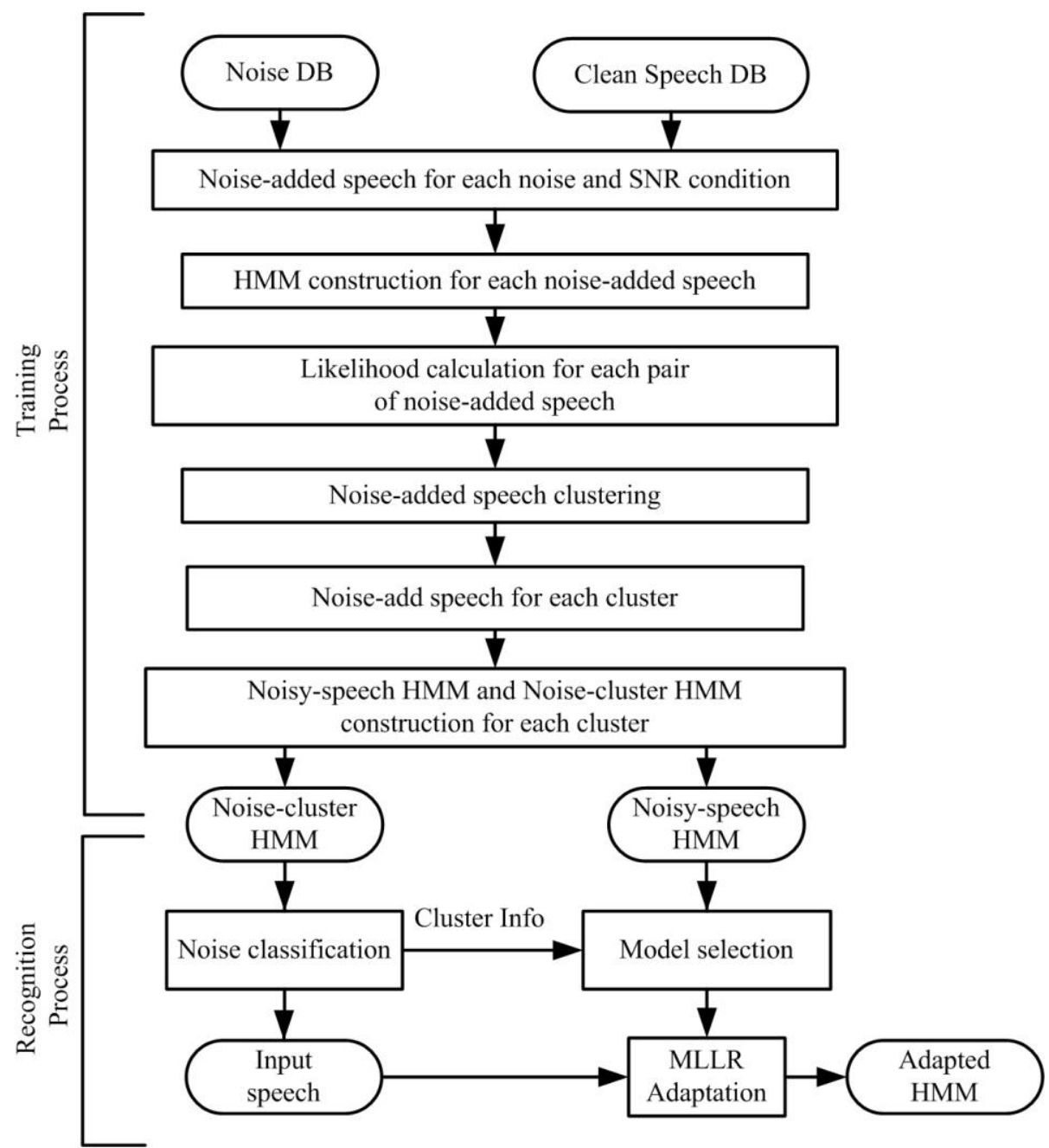

Fig. 1: Piecewise linear transformation for HMM noise adaptation.

There are variations of the proposed method. The first issue is whether the input speech is included in the adaptation set. An input speech with poor quality may degrade the efficiency of adapted acoustic model. Therefore, we evaluate both systems excluding (supervised adaptation) and including the input speech (semi-supervised adaptation). This issue is explained in Section 3.2. Furthermore, due to the variation of speech quality and noisy environment, unsupervised adaptation does not always improve the model quality. In some cases, the original acoustic model is even better than the adapted model. Thus, we also consider the selection between the result provided by the adapted model and that of the unadapted model. Section 3.3 describes this selection procedure. 
It should be noted that the MLLR is used in all adaptation process, not only during the clustering but also in the recognition process. This allows sharing parameters among all distributions in the system, hence reducing the size of the overall system. Consequently, this also reduces the required size of data to be used in the adaptation process.

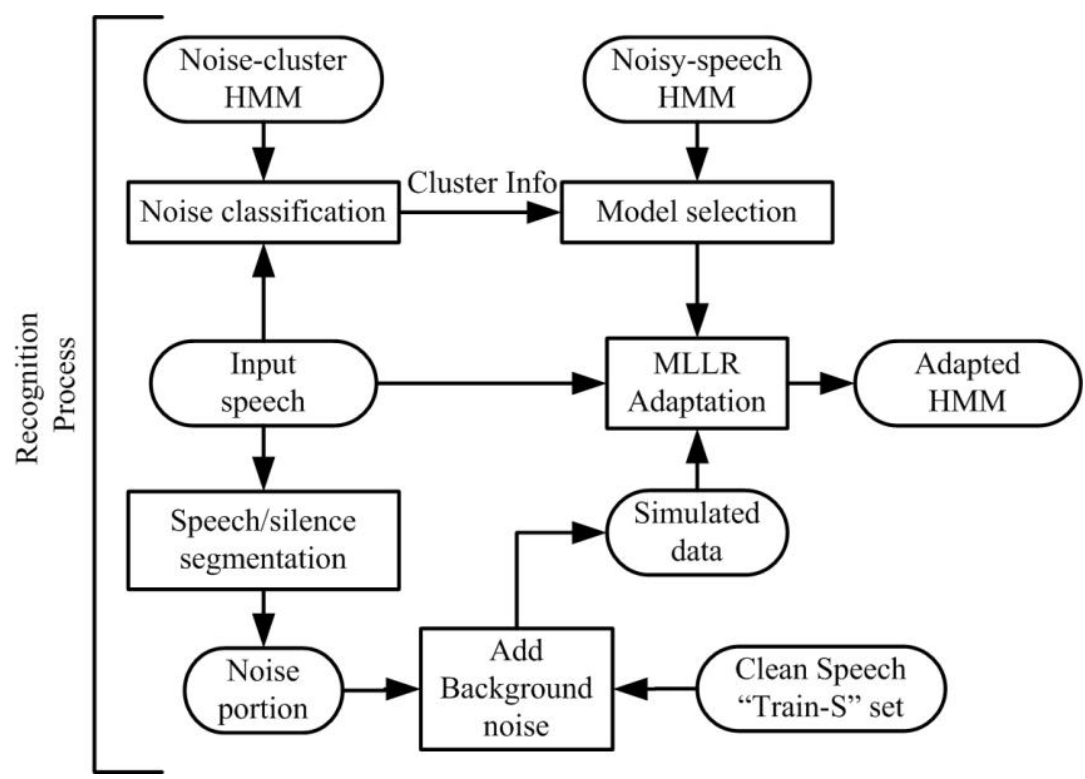

Fig. 2: Recognition process in piecewise linear transformation with simulated-data adaptation for HMM noise adaptation.

\subsection{Simulated-data adaptation}

\subsubsection{Speech/silence segmentation}

Simulated-data adaptation begins with identifying silence parts in the input signal. The silence parts are supposed to be background noise of the current input signal. For our task of isolatedword recognition, we assume that there are short periods of silence at the beginning and the end of the input signal. A hidden Markov model (HMM) is used to segment the input signal into speech and silence portions. Two HMM architectures used for noise extraction. The first algorithm utilizes phone-based HMMs, where 64 HMMs of Thai phonemes including a special phoneme of silence "sil", as shown in Table 1, form an isolated-word recognizer. Figure 3(a) illustrates this HMM structure. The second noise extraction algorithm is based on speech/nonspeech detection. Two states HMM, symbolized with speech and silence, are included in the module as shown in Figure 3(b). In both algorithms, noise portions are the signal regions labeled with silence "sil".

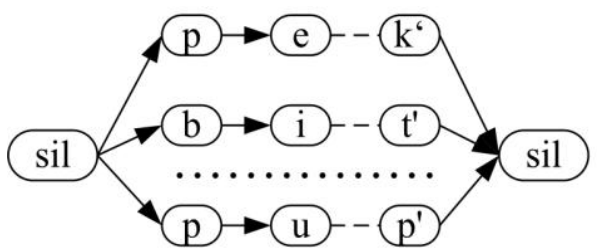

(a) phone-base HMM

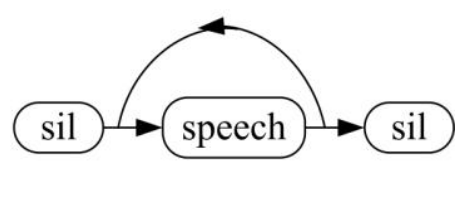

(b) speech/non-speech

Fig. 3: Two HMM architectures used for noise extraction. 
Table 1: 64 Thai phonemes.

\begin{tabular}{|c|c|}
\hline Type & IPA symbol \\
\hline Initial consonant & $\begin{array}{l}, \tau, \chi, \kappa, /, \pi^{\eta}, \tau^{\eta}, \chi^{\eta}, \kappa^{\eta}, \eta, \beta, \delta, \mu, \nu, N, \lambda, \rho, \phi, \sigma, \eta, \omega, \varphi, \pi \rho, \pi \lambda, \\
\pi^{\eta} \lambda, \tau \rho, \tau^{\eta} \rho, \kappa \rho, \kappa \lambda, \kappa \omega, \kappa^{\eta} \rho, \kappa^{\eta} \lambda, \kappa^{\eta} \omega, \phi \rho\end{array}$ \\
\hline Vowel & $, \imath\rceil, \eta, \eta\rceil, v, v\rceil, \varepsilon, \varepsilon\rceil, \in, \in\rceil, \mathrm{o}, \mathrm{o}\rceil, \Theta, \Theta\rceil, \alpha, \alpha\rceil, \square\rceil, \imath\rceil \alpha),\rceil \alpha, v\rceil \alpha$ \\
\hline Final consonant & $\ni, \tau \ni, \kappa \ni, \mu \ni, \nu, N_{\ni}, \sigma \ni, \omega \ni, \varphi \ni$ \\
\hline Silence & $\sigma \imath \lambda$ \\
\hline
\end{tabular}

In both algorithms, HMMs are composed of 16 Gaussian mixtures per state and were trained by the Baum-Welch algorithm. It is noted that the former algorithm gives better noise-region labeling performance with a drawback of computational demand comparing to the latter algorithm.

\subsubsection{Adding background noise}

Given noise portions extracted from the input signal, several issues need to be considered in adding background noise to the pre-recorded clean speech. First we concatenate noise portions extracted from the input signal. There are two noise-only regions in the input signal, at the beginning and at the end of the signal as shown in Figure 4. These noise portions are duplicated and concatenated so that the duration of noise signal is equal to the duration of clean-speech being added. It is noted that simply concatenating noise portions causes an unusual spectral change. However, in this paper, we discard spectral smoothing in order to save processing time.

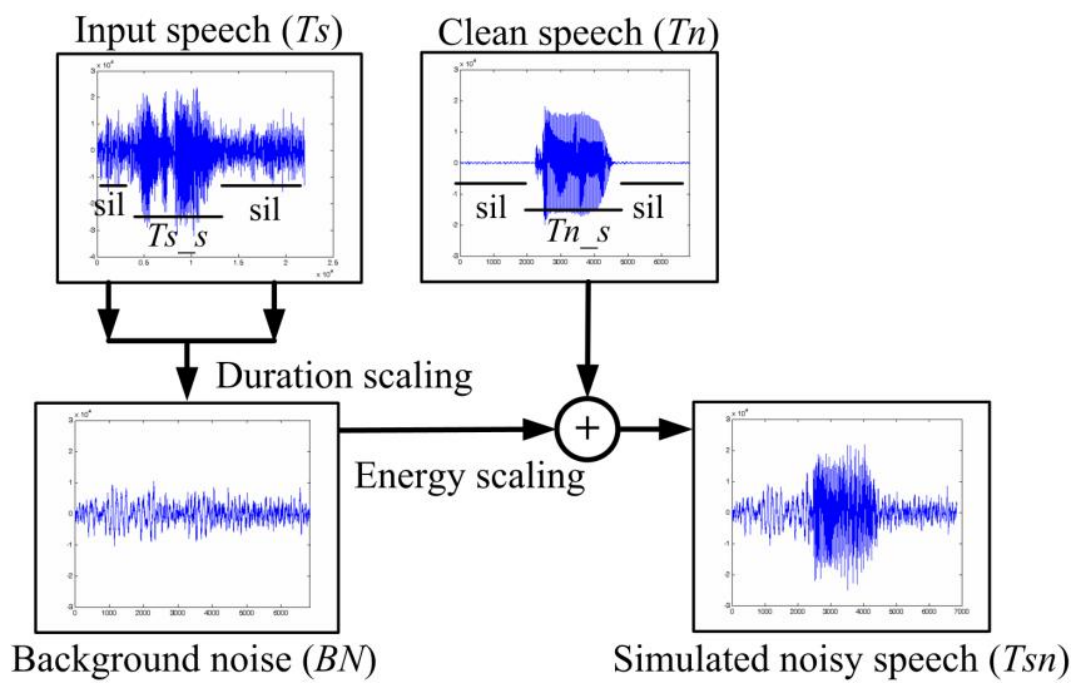

Fig. 4: Adding background noise.

Second, simulated speech for adaptation should have a similar SNR to the input speech. However, estimation of SNR is not trivial and remains unsolved. In this work, we propose a simple way of signal-energy scaling. Let "Train-S" be a set of pre-recorded clean speech, of which correct transcriptions are known. We denote by $T n$ and $T s$ the current input signal and a 
clean speech in the Train-S set. $T n_{-} s_{i}(i=1, \ldots, L n)$ and $T s_{-} s_{i}(i=1, \ldots ., L s)$ is the speech portion of $T n$ and $T s$ and Tn_sil and Ts_sil is the silence portion of Tn and Ts. First, a scale_factor is calculated as follows:

$$
\begin{gathered}
E n g C=\frac{\sum_{i=1}^{L s}\left|T s_{-} s_{i}\right|}{L s} \\
\text { EngS }=\frac{\sum_{i=1}^{L n}\left|T n_{-} s_{i}\right|}{L n} \\
\text { scale_factor }=\frac{\text { EngC }}{E n g S}
\end{gathered}
$$

where EngC and EngS is the energy of $T s \_s$ and $T n \_s$ respectively. Next, the background noise, $B N$, is multiplied by the scale_factor and added to $T s$, resulting a simulated noisy-speech $T s n$.

$$
T s n=B N * \text { scale_factor }+T s
$$

This signal Tsn is included in the adaptation set.

\subsection{Supervised and semi-supervised adaptation}

In the model adaptation process, the MLLR algorithm is applied to the selected HMM with data obtained from the input speech and/or the simulated-data. In order to use the input speech for adaptation, we need its phoneme label. However, the true label of the current input speech in unknown. Therefore, we relied on the label transcribed in the first pass by a selected HMM. The use of input speech with a label automatically transcribed is called an unsupervised adaptation process. In contrast to the use of simulated-data, the noise portion extracted in the process of speech/silence segmentation is added to pre-recorded clean-speech signals $T s$, whose phoneme labels are known. Hence, adaptation using simulated-data is supervised. An adaptation process that uses both the input speech and a set of simulated-data is denoted as semi-supervised adaptation.

\subsection{Recognition result selection}

Theoretically, an adapted acoustic model should enhance the recognition accuracy. However, this assertion does not always hold in practice, due to many factors such as the recording condition and the speaking style. In the case where the quality of adaptation data is low, the recognition result given by the un-adapted HMM is useful.

In this work, we investigate 2 recognition schemes: one-step and two-step methods. The former method always relies on the result provided by the adapted model. The latter method chooses the final result from either the result of the un-adapted HMM or that of the adapted HMM. Indeed, the result whose likelihood, compared to the corresponding model, is higher is selected as final result. Figure 5 shows these 2 selection methods used in this work.

Table 2 summarizes the different settings of the proposed S-PLT system investigated in this work. Certainly, other parameters like the number of words in Train-S or the number of clusters in MLLR adaptation also has influence on the accuracy of the system. These parameters will also be investigated experimentally in Section 5 . 
Table 2: Four configurations of the proposed S-PLT system which will be investigated in this paper.

\begin{tabular}{ll}
\hline Method & Description \\
\hline Method1-1 & One-Step method with supervised MLLR \\
Method1-2 & One-Step method with semi-supervised MLLR \\
Method2-1 & Two-Step method with supervised MLLR \\
Method2-2 & Two-Step method with semi-supervised MLLR \\
\hline
\end{tabular}
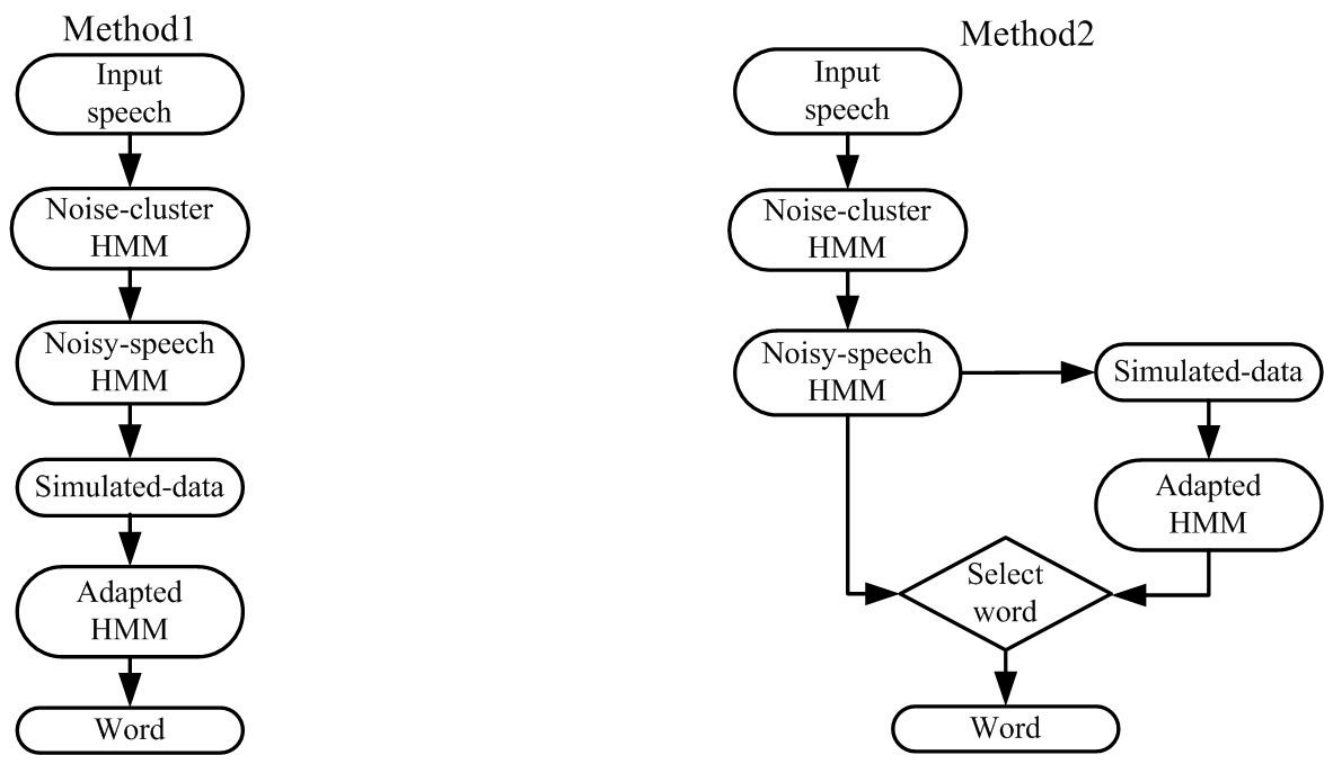

Fig. 5: The two selection methods used in the S-PLT.

\subsection{Using simulated-data adaptation in the normal MLLR process}

It is noted that the idea of simulated-data adaptation is to increase the number of adaptation data by adding the noise portion extracted from an incoming input speech to a set of pre-recorded clean speech. Therefore, it can be used in any adaptation algorithm such as the general MLLR process, where an original acoustic model is adapted with an incoming input speech. MLLR with simulated-data adaptation is named shortly as S-MLLR. S-MLLR can be performed in 4 variations, S-MLLR1-1, S-MLLR1-2, S-MLLR2-1, and S-MLLR2-2, similar to S-PLT described in the previous section.

\section{EXPERIMENTAL SETTING}

\subsection{Task}

This work concerns the isolated-word recognition. Our system is a phoneme-based with HMMs representing 64 Thai phones. These allow cover up all phones from the NECTEC-ATR corpus 
[14] which is used throughout this work. Each monophone HMM consisted of 5 states and 16 Gaussian mixtures per state. 39 dimensional vectors (12 MFCC, 1 log-energy, and their first and second derivatives) were used as recognition features.

The baseline acoustic model was clean monophone HMM. It was trained by using phoneticallybalanced read by 16 male and 16 female speakers. The total number of training utterances was 32,000 .

In all experiments, the clean speech files were taken from the NECTEC-ATR.

\subsection{Noise data for training}

Eight kinds of noise from JEIDA (Japan Electronic Industry Development Association) [12], including crowded street, machinery factory, railway station, large air-condition, trunk road, elevator, exhibition in a booth, and ordinary train, 1 large-size car noise from NOISEX-92 [13] were used for noise clustering. All noises from JEIDA and NOISEX-92 as well as the clean speech from NECTEC-ATR were preprocessed by reducing the sampling rate to $8 \mathrm{kHz}$. Noisy speech was prepared by adding the noise from JEIDA or NOISEX-92 to the clean speech of NECTEC-ATR at various SNR $(0,10$ and $15 \mathrm{~dB})$. A noise HMM with 16 mixtures was trained for each noise by the Baum-Welch algorithm.

\subsection{Noise data for testing}

Two test sets, Test- 1 and Test-2, were used to evaluate the proposed method.

"Test-1" contained 3200 words uttered by 5 male speakers. Two noises, "computer room" (Noise1) from JEIDA and "exhibition" (Noise2) recorded over four days in March of 2005 at exhibition of NSTDA Annual Conference S\&T in Thailand 2005, were digitally added to the utterances at three SNR levels: 0,10 and $15 \mathrm{~dB}$. These are new noises which differ from the 9 noise samples used to train the system.

"Test-2" contained 76 words utterances from 50 speakers collected over four days in August of 2005 in the actual environment of "exhibition" at ICTEXPO 2005 in Thailand. The noise power was estimated using the noise periods immediately before and after each sentence utterance. The power of noise-added speech was estimated as the mean value averaged over the utterance period. Based on these values, the estimated SNR was 5-0 dB. This task was difficult, since the noise was non-stationary.

\subsection{Data for supervised adaptation}

In order to constitute the Train-S set for model adaptation, several criteria are used to select speakers and lexical words from the NECTEC-ATR corpus. Speakers used in Train-S are selected from speakers in the training set, not from any test set.

For speaker selection, we limited to male speakers with clear speech. For this criterion, four speakers "M1", "M2", "M3", and "M4" were selected. These speakers were also used to test the effect of the number of speakers on the adaptation process. For this test we denote by "MIX" the set of data containing all of these 4 speakers.

Next, we choose the set of words to be used in Train-S. Two criteria were considered. First, these words should be correctly recognized by clean model and second these words should cover all 64 phones present in the system. According to these criteria, 22 words were selected for model adaptation.

Moreover, in order to investigate the effect of phones in Train-S on the adaptation procedure, we 2 additional subsets were constructed from these 22 words. The first subset contained 8 words which cover 41 phones and the second subset contained 16 words with 56 phones. 


\section{EXPERIMENTAL RESULTS}

\subsection{Comparison of four methods of simulated-data adaptation}

The first experiment aims at comparing four methods using simulated-data adaptation as described in Table 2. The four methods can be applied to both S-MLLR and S-PLT models. In this experiment, the set of pre-recorded clean speech Train-S contained speeches from one speaker (M1) and covers 22 distinct words. The number of clusters used in the MLLR process was set to 1 .

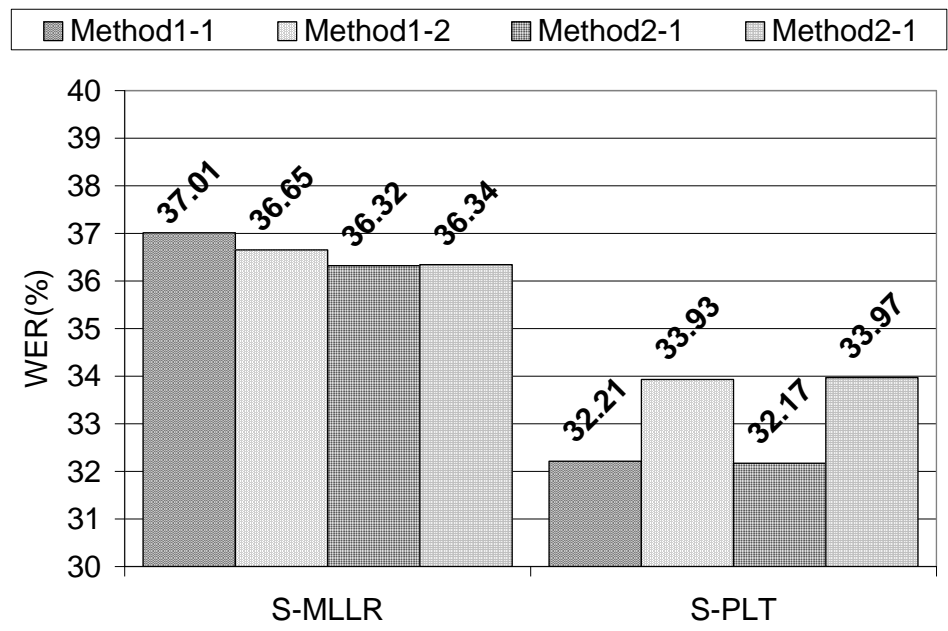

Fig. 6: Comparison of S-MLLR and S-PLT on Test-1 data (Noise 1 noise-added speech, SNR: $15 \mathrm{~dB}, 10 \mathrm{~dB}$ and $0 \mathrm{~dB})$.

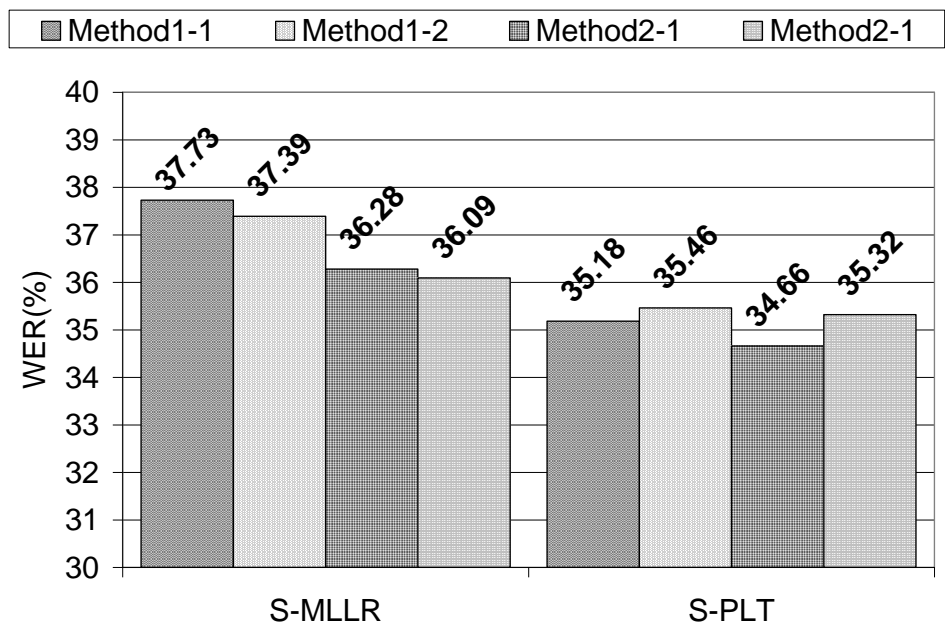

Fig. 7: Comparison of S-MLLR and S-PLT on Test-1 data (Noise 2 noise-added speech, SNR: $15 \mathrm{~dB}, 10 \mathrm{~dB}$ and $0 \mathrm{~dB}$ ).

Figures 6 and 7 show word error rate (WER) results of Test-1 for 2 types of noises, Noise 1 and Noise2. The results consistently present the better performance of S-PLT over the simple S- 
MLLR. For S-PLT, the best configuration is the "Method2-1", where the adaptation set excludes the input speech and the recognition result is selected between results of the original and adapted acoustic models. The most important reason that the adaptation set should exclude the input speech is that when the quality of input speech is poor, the original acoustic model will produce wrong transcription of the input speech. Using the wrong transcription in the adaptation process yields a distorted acoustic model. Though the input speech has a low quality, the noiseonly portion in the speech signal is a good source for simulating adaptation data from prerecorded clean speeches in which we know their correct transcriptions.

\subsection{Experiments on the number of MLLR clusters and the size of adaptation data}

In this subsection, the number of MLLR clusters and the size of adaptation data were optimized for the S-PLT model. Train-S was the same as that used in the previous subsection, but the number of MLLR clusters varied between 1, 8, and 16, while the number of words covered was set to 8,16 , and 22 , spanning over 41,56 , and 64 phonemes respectively.

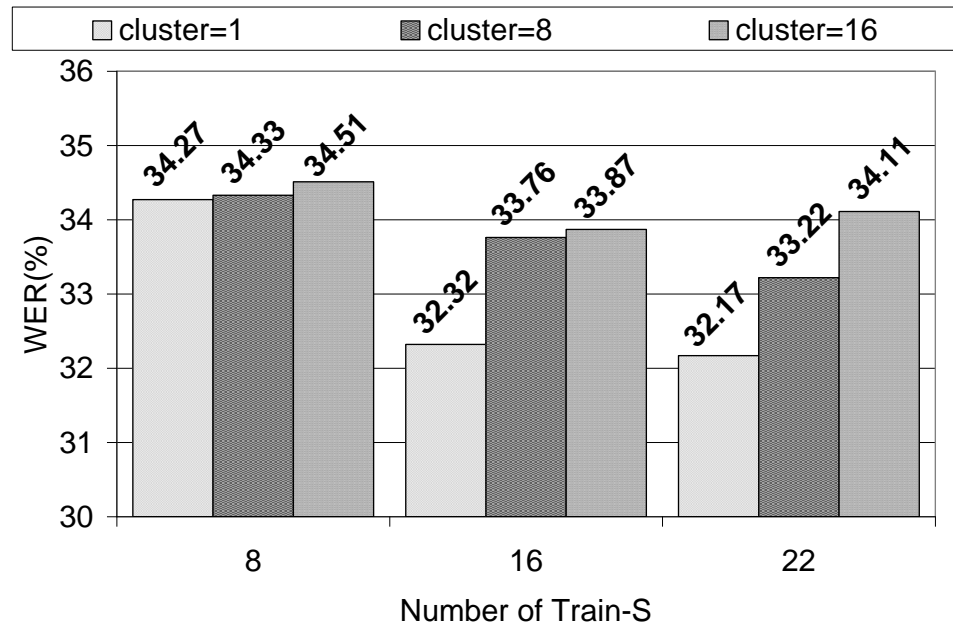

Fig. 8: Recognition results using S-PLT by "Method2-1" on Test-1 data. (Noise 1 noise-added speech, SNR: $15 \mathrm{~dB}, 10 \mathrm{~dB}$ and $0 \mathrm{~dB}$ ).

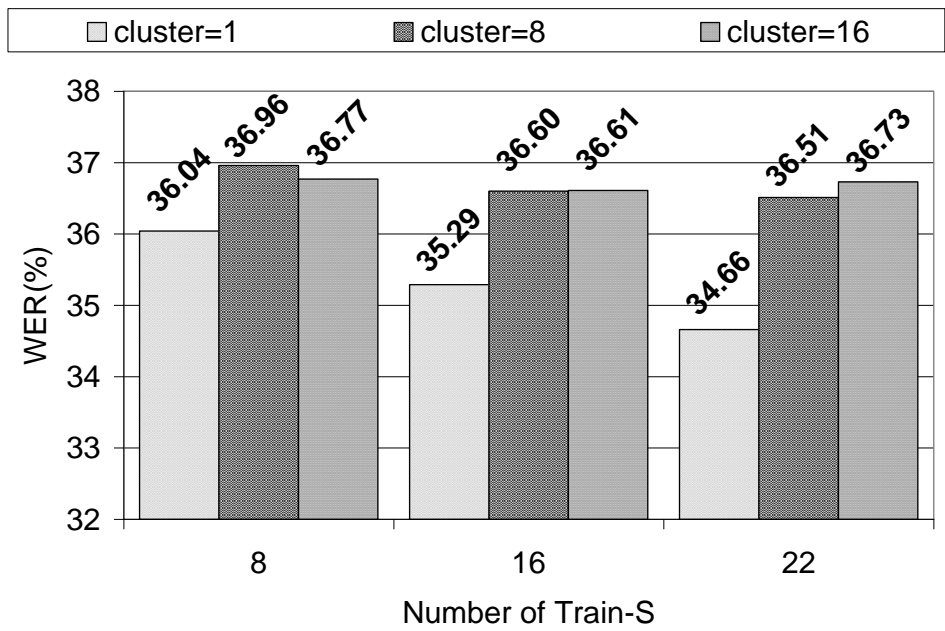

Fig. 9: Recognition results using S-PLT by "Method2-1" on Test-1 data (Noise 2 noise-added speech, SNR: $15 \mathrm{~dB}, 10 \mathrm{~dB}$ and $0 \mathrm{~dB}$ ). 
Figures 8 and 9 show evaluation results of Test-1 for the noises Noise1 and Noise2. Both figures obviously show that one MLLR cluster achieves the best performance in every case of the size of Train-S and the type of noises. The more the number of words covered in Train-S is used, the lower WER is achieved. However, we limit the largest number of words in Train-S to 22, which covers all phonemes appearing in the evaluation task. A larger set of Train-S may produce a higher accuracy, but increases computational time.

\subsection{Experiments on different speakers used in simulated-data adaptation}

In the case that speeches from only one speaker are included in the simulated adaptation data, increasing the size of adaptation data tends to produce a speaker-dependent acoustic model. Using the speaker-specific acoustic model may reduce the system accuracy when evaluating with speeches from various speakers. Therefore, in this subsection, five experiments on S-PLT were performed to explore this phenomenon. Each of the first four experiments uses speeches of only one speaker (M1 to M4). Randomly mixed speeches from M1 to M4 speakers were used in the last experiment, denoted as MIX. The number of MLLR cluster was set to 1.

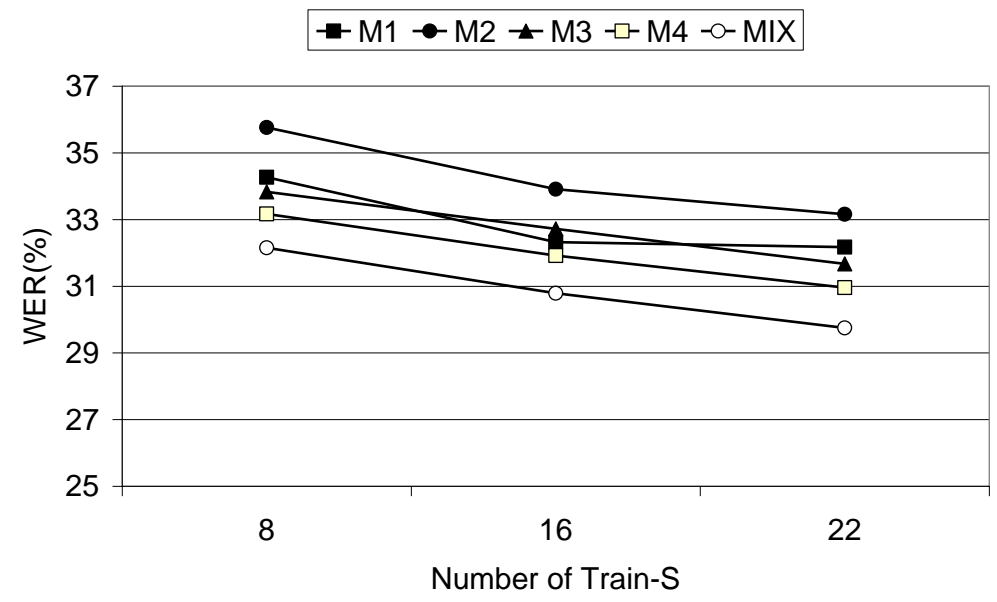

Fig. 10: Recognition results using S-PLT by "Method2-1" on Test-1 data (Noise 1 noise-added speech, SNR: $15 \mathrm{~dB}, 10 \mathrm{~dB}$ and $0 \mathrm{~dB}$ ).

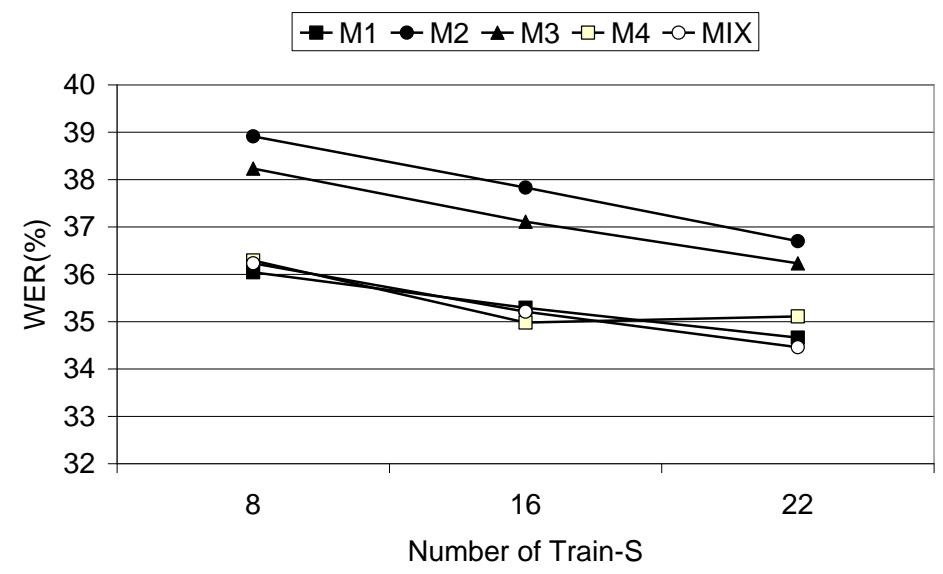

Fig. 11: Recognition results using S-PLT by "Method2-1" on Test-1 data (Noise 2 noise-added speech, SNR: $15 \mathrm{~dB}, 10 \mathrm{~dB}$ and $0 \mathrm{~dB}$ ). 
Figures 10 and 11 plot results of Test- 1 for Noise 1 and Noise 2 respectively. According to results, WER is reduced as the size of adaptation data increases. We conclude that the phenomenon of speaker-mismatching is not significant even when the largest set of adaptation data is conducted. The MIX case, where the adaptation set contains speeches from various speakers is obviously better than the use of one specific speaker model. This indicates the original acoustic model should be adapted with its speaker independent property maintained.

\subsection{Comparison with conventional methods}

In this subsection, our methods of using simulated-data adaptation, S-MLLR and S-PLT, at their best configurations were compared to several conventional methods including the baseline system (without any adaptation process), MLLR, model selection, and PLT. "Train-S" consisted of speeches covering 22 words from mixed speakers and the number of MLLR cluster was 1. Evaluation results on "Test-1" with the noises "Noise-1" and "Noise-2" are expressed in Figure 12 and 13 respectively. Results obviously show high improvement of both the S-MLLR and SPLT over the other methods.

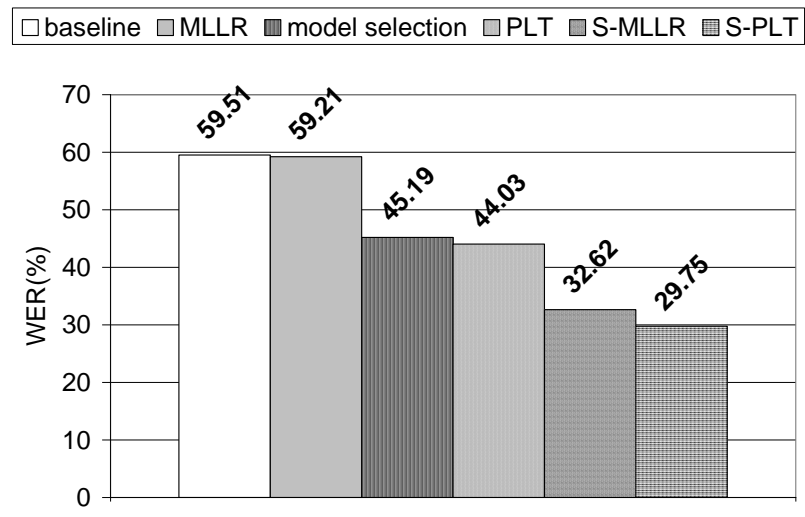

Fig. 12: Comparison of baseline, MLLR, model selection and PLT using tree-structured clusters, S-MLLR and S-PLT by "Method2-1" on Test-1 data (Noise 1 noise-added speech, Train-S: Mix, SNR: 15, 10 and $0 \mathrm{~dB}$ ).

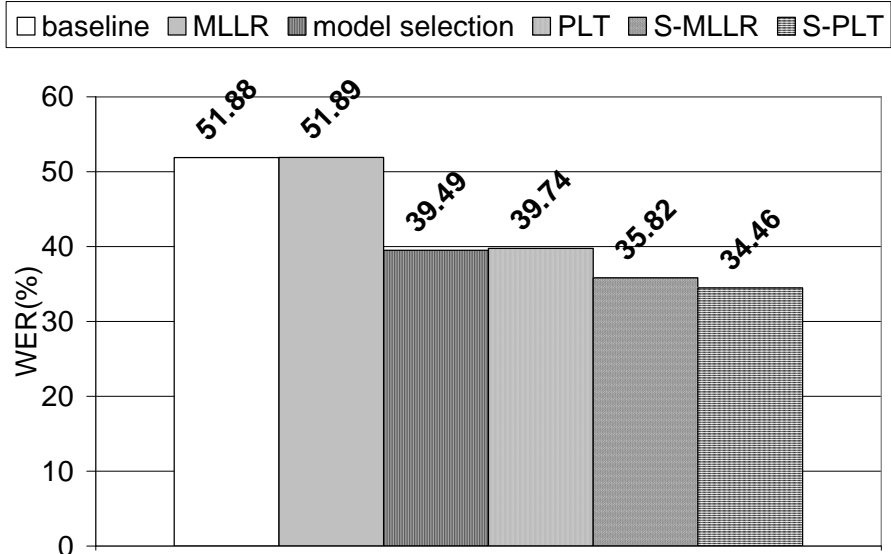

Fig. 13: Comparison of baseline, MLLR, model selection and PLT using tree-structured clusters, S-MLLR and S-PLT by "Method2-1" on Test-1 data (Noise 2 noise-added speech, Train-S: Mix, SNR: 15, 10 and $0 \mathrm{~dB}$ ). 


\subsection{Experiments on real noisy speech (Test-2)}

The last experiment consisted in testing our proposed methods on the "Test-2" set. All parameters were the same as those set in the previous subsection. Results, shown in Figure 14, indicate the advantage of our methods on the real noisy speech. The best performance is by SPLT, which achieves relatively $21.4 \%$ WER reduction from the normal PLT method.

\section{$\square$ baseline $\square$ MLLR $\square$ model Selection $\square$ PLT $\square$ S-MLLR ⿴囗大 S-PLT}

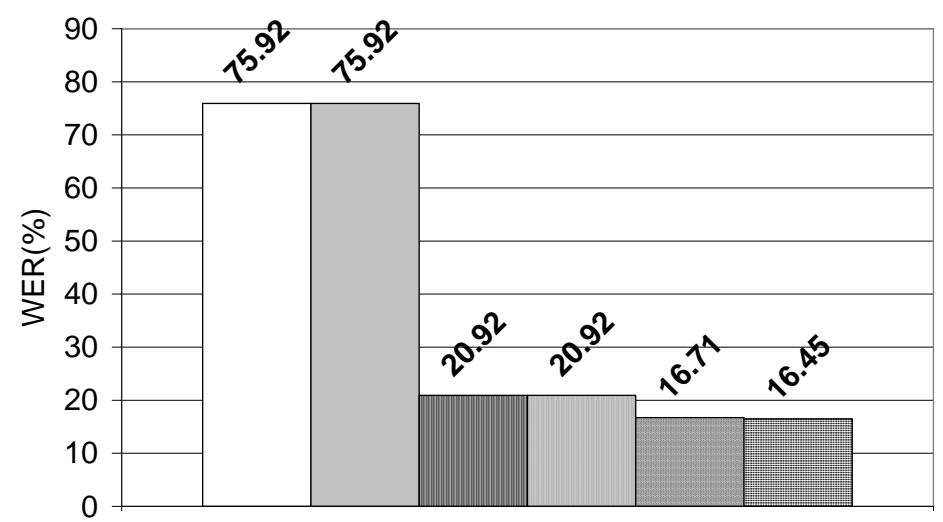

Fig. 14: Comparison of baseline, MLLR, model selection using tree-structured clusters, PLT using tree-structured clusters, S-MLLR by "Method2-1" and S-PLT by "Method2-1" on Test-2 data. (Train-S: Mix).

\section{CONCLUSION AND FUTURE WORKS}

This paper proposed a new idea of using simulated-data adaptation in piecewise linear transformation (PLT). The idea is to increase the number of adaptation data used in PLT by adding a noise portion from an input speech to a set of clean speech. The experiment showed that the best performance was achieved when the set of clean speech contained as many distinctive phonemes and speaker as possible. The use of Method2-1, where a recognition results was selected from two results produced by the general acoustic model and the adapted acoustic model achieved better performance than using only the adapted acoustic model. The experiment also proved the advantage of using simulated-data adaptation even in the simple MLLR (S-MLLR).

Future works include an evaluation of the idea by a larger set of speech from real environments and in various situations. Further improvement of noise addition in the simulated-data adaptation will also be investigated. Another interesting issue is that the selection of clean speech from different speakers might affect the recognition result. Selecting a set of speakers that best match the input speech might give the better performance. This issue will be explored.

\section{ACKNOWLEDGEMENT}

A special thank is given to Dr. Zhipeng Zhang for his valuable advice. 


\section{REFERENCES}

1. Gales, M.J.F. (1995), Model-based techniques for noise robust speech recognition, $\mathrm{PhD}$ thesis University of Cambridge.

2. Leggetter, C.J. and Woodland, P.C. (1995), Maximum likelihood linear regression for speaker adaptation of continuous density HMMs, Computer Speech Language, vol. 9, pp. 171-186.

3. Gales, M.J.F. and Woodland, P.C. (1996), Mean and variance adaptation within the MLLR framework, Computer Speech Language, vol. 10, pp. 249-264.

4. Gauvain, J.L. and Lee, C.H. (1994), Maximuma posteriori estimation for multivariate Gaussian mixture observations of Markov chains, IEEE Trans. Speech Audio Processing, vol. 2, pp. 291-298.

5. Chesta, C., Siohan, O., and Lee, C.-H. (1999), Maximum a posteriori linear regression for hidden Markov model adaptation, Proc. EuroSpeech, pp. 211-214.

6. Gales, M.J.F. and Young, S. (1992), An improved approach to the hidden Markov model decomposition of speech and noise, Proc. ICASSP, pp. 233-236.

7. Martin, F., Shikano, K., and Minami, Y. (1993), Recognition of noisy speech by composition of hidden Markov models, Proc. Eurospeech, pp. 1031-1034.

8. Minami, Y. and Furui, S. (1995), A maximum likelihood procedure for a universal adaptation method based on HMM composition, Proc. ICASSP, pp. 129-132.

9. Zhang, Z.P. and Furui, S. (2004), Piecewise-linear transformation-based HMM adaptation for noisy speech, Speech Communication, vol. 42, no. 1, pp. 43-58.

10. Zhang, Z.P. and Furui, S. (2005), Tree-Structured Clustering Methods for Piecewise Linear transformation-Based Noise Adaptation, IEICE TRANS. INF. \& SYST., vol. E88-D, no. 9, pp. 2168-2176.

11. Kosaka, T., Matsunaga, S., and Sagayama, S. (1996), Speaker-independent speech recognition based on tree-structured speaker clustering, Computer Speech Language, vol. 10 , pp. 55-74.

12. http://www.milab.is.tsukuba.ac.jp/corpus/noise db.html

13. http://www.speech.cs.cmu.edu/comp.speech/ Section1/ Data/noisex.html

14. Kasuriya, S., Sornlertlamvanich, V., Cotsomrong, P., Jitsuhiro, T., Kikui, G., and Sagisaka Y. (2003), NECTEC-ATR Thai speech corpus, Proc. of Oriental COCOSDA2003, pp. 105111. 